\title{
Cáncer prostático incidental en biopsias obtenidas por resección transuretral de próstata con diagnóstico de hiperplasia prostática benigna.
}

\author{
Sara Rodríguez-Carrillo ${ }^{1 *}$. Efrén Rafael Ríos-Burgueño². Ana Karen Soto-Sañudo3. Ana cristina Bení- \\ tez-Morales ${ }^{4}$. \\ ${ }^{1}$ Residente de tercer año de Anatomía Patológica en Hospital Civil de Culiacán. \\ ${ }^{2}$ Médico Anatomo patólogo, jefe de curso y del servicio de Anatomía Patológica en el Hospital Civil de Cu- \\ liacán. \\ ${ }^{3}$ Residente de Segundo año de Anatomía Patológica en Hospital Civil de Culiacán. \\ ${ }^{4}$ Residente de Segundo año de Anatomía Patológica en Hospital Civil de Culiacán.
}

Recibido 13 de Enero 2016, aceptado 23 de Marzo 2016

\section{RESUMEN}

OBJETIVOS: Determinar la frecuencia de cáncer de próstata incidental en muestras con diagnóstico clínico de Hiperplasia Prostática Benigna. MATERIAL Y MÉTODOS: Estudio de corte transversal. Se incluyeron biopsias obtenidas por la técnica quirúrgica de resección transuretral de próstata (RTUP), con diagnóstico de Hiperplasia Prostática Benigna (HPB). Se recabaron muestras que fueron diagnosticadas incidentalmente con Cáncer prostático en un periodo de 2010 a 2014 . RESULTADOS: Se obtuvieron 233 biopsias por RTUP en pacientes con rango de edad de 48 a 91 años con diagnóstico clínico de Hiperplasia de Próstata Benigna; 19 de estas muestras se diagnosticaron como Adenocarcinoma de próstata, que corresponde al $8.1 \%$ y se categoriza como incidental. CoNCLUSIONES: Este estudio comprueba el bajo porcentaje de cáncer prostático incidental, sin embargo estos datos sugieren que los hombres considerados para este manejo quirúrgico de la Hiperplasia de Próstata Benigna debería ser informado sobre este hallazgo anatomopatológico y que esté puede ser albergado sin ser detectado clínicamente.

Palabras claves: hiperplasia prostática benigna, cáncer de próstata incidental, resección transuretral.

OBJECTIVES: To determine the frequency of incidental prostate cancer samples diagnosed with Benign Prostatic Hyperplasia. MATERIAL AND METHODS: Cross-sectional study biopsies obtained by surgical technique transurethral resection of the prostate (TURP), diagnosed with benign prostatic hyperplasia (BPH) were included. Samples diagnosed with prostate cancer incidentally in a period from 2010 to 2014 were collected. RESULTS: 233 TURP biopsies were obtained in patients with age range of 48 to 91 years with a clinical diagnosis of benign prostatic hyperplasia; 19 of these samples were diagnosed as prostatic adenocarcinoma, which corresponds to $8.1 \%$ and is categorized as incidental. CONCLUSIONS: This study tests the low percentage of incidental prostate cancer, however, these data suggest that men considered for this surgical management of Benign Prostatic Hyperplasia should be informed of this pathological finding and it can be harbored without being detected clinically.

KEYWORDS: benign prostatic hyperplasia, incidental prostate cancer, transurethral resection.

\section{Introducción}

El cáncer de próstata es una de las neoplasias malignas más frecuente en los hombres y se incrementa conforme aumenta la edad. El $87.6 \%$ de las defunciones se registraron en la población mayor de 65 años. ${ }^{1}$

\footnotetext{
${ }^{*}$ Correspondencia: Sara Rodríguez Carrillo. zharaly31@gmail.com Eustaquio Buelna No. 91. Colonia Gabriel Leyva; C.P.80030. Culiacán, Sinaloa.
}

En México, las neoplasias malignas ocuparon el segundo lugar en la tabla de mortalidad general. El cáncer de próstata fue la segunda neoplasia más frecuente en el hombre en edad postreproductiva, con 3766 defunciones y tasa de 71.1 por 100 mil en el grupo de 65 años y más, por lo que se considera que es un cáncer hormonodependiente. 
En estudios sobre autopsias se ha encontrado que el $10.6 \%$ en los varones de 50 a 59 años, el $43.6 \%$ entre los 80 y 89 años y el $83 \%$ de los varones de 90 a 99 años tienen cáncer de próstata. ${ }^{1}$ La tasa de mortalidad por este tipo de cáncer ha disminuido en los Estados Unidos, esto se ha atribuido al diagnóstico precoz. Los pacientes diagnosticados en estado precoz tienen probablemente mejor pronóstico que los diagnosticados en estado avanzado. ${ }^{2}$

La literatura internacional refiere que $4 \%$ a $20 \%$, (con una media de $10 \%$ ) de los pacientes intervenidos de Hiperplasia Prostática Benigna (HPB) se les detecta Cáncer de Próstata Incidental (CPI) en el reporte histopatológico. Un estudio publicado a principios del 2008 refiere que la prevalencia de Cáncer en población sana de donadores cadavéricos fue del $12 \% .{ }^{10}$

El estudio de la próstata en productos de resección transuretral (RTU) fue el método utilizado con más frecuencia durante décadas en el estudio de la biopsia prostática. Por lo general se empleaba en pacientes que presentaban obstrucción por hiperplasia nodular o por carcinomas que habían crecido lo suficiente para causar síntomas obstructivos.

El cáncer incidental de próstata (CAI) se puede definir como aquel que cursa sin evidentes síntomas neoplásicos a este nivel; que es descubierto causalmente en el examen microscópico del tejido resecado posterior a una cirugía prostática, bajo el supuesto diagnóstico de benignidad. Es una forma de cáncer prostático que no tiene signifi- cado clínico en el paciente en el que fue descubierto. Por lo tanto, aparentemente existe sólo un pequeño porcentaje de cánceres que progresan y se diseminan. El porqué de esta agresividad selectiva se desconoce por completo. ${ }^{3-4}$

No está totalmente establecido cuál debe ser la conducta más adecuada ante el hallazgo de un cáncer de próstata incidental. ${ }^{5} \mathrm{La}$ prostatectomía radical o la radioterapia, probablemente suponga sobretratamiento en un porcentaje importante de pacientes, mientras que la observación y seguimiento conlleva un riesgo de progresión que no podemos predecir. ${ }^{6-7}$

\section{Material y métodos}

Se trata de un estudio descriptivo de corte transversal retrospectivo, se incluyen todas las biopsias de próstata con diagnóstico clínico de Hiperplasia de Próstata Benigna previo a procedimiento quirúrgico, sin diagnóstico de cáncer de próstata previo; en el período comprendido del primero de Junio 2010 a primero de Junio de 2014 en el Departamento de Anatomía Patológica del Hospital Civil de Culiacán Sinaloa.

Se excluyeron productos de prostatectomía y biopsias transrectales de próstata. Se realizó estadística descriptiva y se estimaron intervalos de confianza del 95\%. Este es una investigación sin riesgo para los pacientes, la muestra de la población a estudiar fueron tejidos almacenados en el departamento de Anatomía Patológica del Hospital Civil de Culiacán, sin tener contacto directo con los pacientes. 


\section{Resultados}

En el periodo de Junio 2010 - Junio 2014, se revisaron un total de 233 laminillas teñidas con hematoxilina y eosina de biopsias obtenidas por resección transuretral de próstata de pacientes con un rango de edad 48 y 91 años (media 72) con diagnóstico clínico previo de hiperplasia prostática benigna.

El material recabado por año fue el siguiente: En el año 2010 con un total de 27 muestras (2 adenocarcinomas bien y moderadamente diferenciados respectivamente), en el 2011 con 57 biopsias (4 adenocarcinomas moderadamente diferenciados), para el año 2012 se recabaron 46 muestras (3 adenocarcinomas prostáticos), correspondiente al año 2013 se obtuvieron 77 muestras (6 con diagnóstico de adenocarcinoma de próstata) y en el año 2014 se recaban 43 biopsias (4 con adenocarcinoma, tres como poco diferenciado y uno moderadamente diferenciado). Como resultado final en el periodo de 4 años (2010 2014) se obtiene un total de 19 muestras con diagnóstico final por histología de Adenocarcinoma de próstata con diferentes grados de diferenciación de acuerdo a lo mencionado anteriormente, tal como se muestra en la figura 1.

Esto corresponde al $8.15 \%$ de la población estudiada. En la figura 2., De las 233 muestras, 214 fueron diagnosticadas por sus características histológicas como hiperplasia fibromuscular y adenomatosa, con prostatitis crónica.
Fig. 1. Biopsias por resección transuretral en el periodo 2010-2014 de laboratorio de anatomía patológica del Hospital Civil de Culiacan.

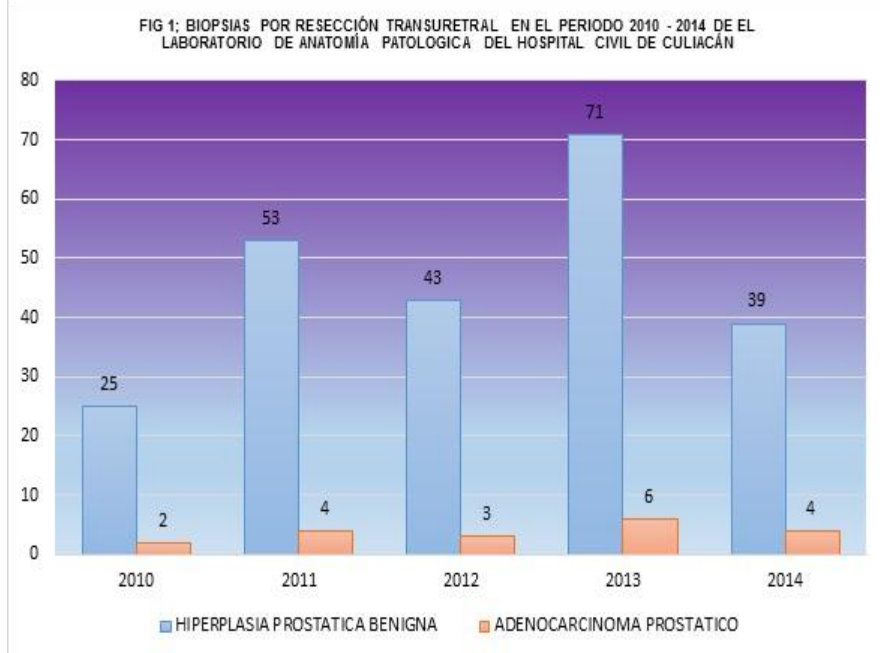

Fig. 2. Porcentaje de adenocarcinoma incidental PORCENTAJE DE ADENOCARCINOMA INCIDENTAL

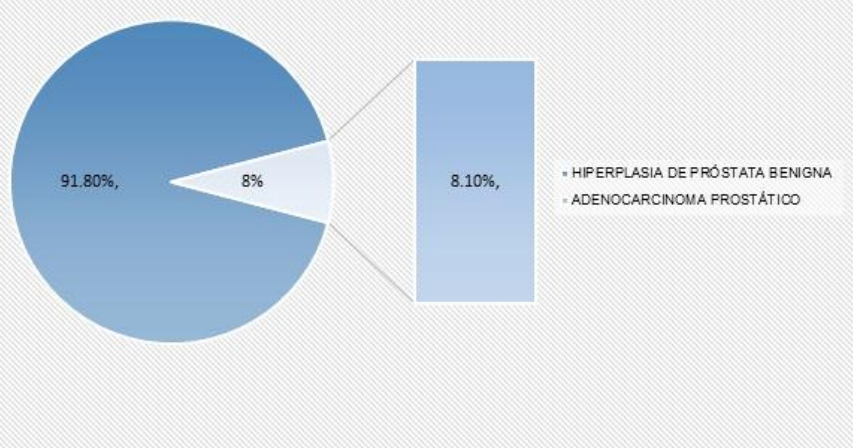

Se observa en el grupo sin Carcinoma prostático incidental, que el mayor número de pacientes se hallan entre 60 a 85 años, que son 187 pacientes $(80.2 \%)$; es semejante en el grupo con CPI donde en el mismo rango de edad es entre los 60 a 85 años, donde se encuentran 19 pacientes (8.1 $\%)$. Es decir que la edad no nos podría indicar que pacientes tienen más probabilidad de tener $\mathrm{CPI}$. 
El Hospital Civil de Culiacán, es una institución de Salud el cual cuenta con el Servicio de Patología, mismo donde se reciben por año de 4000 a 4500 quirúrgicos en promedio, de los cuales el 1.7 \% corresponden a biopsias de próstata. Por año se reciben en un promedio 68 biopsias de próstata.

Cabe mencionar que en este trabajo no se pudo realizar una correlación clínica con los niveles de APE (antígeno prostático específico), por la falta de datos en las solicitudes de dichos estudios y el seguimiento del paciente, esto debido a que el paciente que acude a consulta al Hospital Civil de Culiacán por lo común no es un paciente que se realice controles regulares.

\section{Discusión}

El diagnóstico de cáncer de próstata tras la realización de una RTUP, motivada por un síndrome prostático que no responde al tratamiento médico pautado, es poco frecuente y supone un hallazgo incidental de la neoplasia. Teniendo en cuenta que el tejido glandular que se extrae en esta intervención vendrá de la Zona Transicional de la próstata y que la probabilidad de diagnosticar un cáncer prostático en esta localización no asciende al $25 \%$, el porcentaje de pacientes que se beneficiarán de un diagnóstico histológico mediante este método será escaso. Se estima que el porcentaje de detección de un cáncer de próstata tras RTUP no es insignificante y constituiría hasta el $10 \% .^{8}$ La tasa de cáncer incidental en nuestro estudio fue de $8.1 \%$, las tasas relativas de los cánceres diagnosticados RTUP son similares en otras series de distintas poblaciones de pacientes. Por el contrario estudios anteriores tienen demostrado a la zona periférica como el lugar prominente de la génesis tumoral en el adenocarcinoma de próstata primario. Por lo tanto los cánceres de resección transuretral de próstata diagnosticados pueden representar un grupo de cánceres con una ubicación en el mapeo anatómico distinto, en comparación con los detectados convencionalmente a través de biopsias con aguja de próstata. ${ }^{8}$

En el estudio de Melchior y colaboradores, la tasa de cáncer de próstata incidental fue de 5.4\% (104 de 1931 pacientes). Llegaron a la conclusión de que actualmente no existe posibilidad de predecir con seguridad la ausencia de agresividad de cáncer de próstata después de la RTUP y recomendar por lo tanto de manera segura la observación en lugar de seguir con la terapia. Por lo tanto, los pacientes con cáncer de próstata incidental necesitan ser aconsejados de forma individual. ${ }^{9}$

La mayoría de los hombres con síntomas obstructivos actualmente se tratan con medicamentos o alternativas a la RTUP mínimamente invasivas, tales como terapia láser. ${ }^{10}$ La realización de una resección transuretral de próstata sólo para detectar cáncer no está aceptada como indicación formal, excepto que los pacientes tengan biopsias negativas y refieran síntomas obstructivos que justifiquen la intervención. Al respecto, van Renterghem estudió pacientes asintomáticos con $\mathrm{Hi}$ perplasia de Próstata Benigna por medio de urodinamia detectando obstrucción infravesical en la 
mayoría de ellos, por lo que propuso realizar cirugías diagnósticas con el triple objetivo de aliviar la obstrucción (aún si fuera asintomática), hacer biopsias extensas y disminuir los valores de PSA. ${ }^{11}$

A pesar de que los argumentos científicos que avalan la "RTU diagnóstica" son escasos, las evidencias a su favor van tomando mayor importancia. Entre estas se pueden mencionar:Pacientes asintomáticos con PSA elevado que pueden tener obstrucciones significativas y beneficiarse con la cirugía. ${ }^{11}$ Valores altos de PSA se asocian a riesgo de retención urinaria. ${ }^{12}$

La RTUP tiene baja tasa de complicaciones, que pueden reducirse aún más haciendo resecciones limitadas que eviten la resección apical y la perforación capsular.

Las neoplasia de la zona transicional son generalmente de bajo grado y con menor tendencia a comprometer la cápsula y mestastatizar, por lo que hay buena oportunidad de curación. 13,14

La detección de adenocarcinoma en pacientes operados por hiperplasia oscila entre 4 y $15 \%$ en la literatura. ${ }^{11}$ Según la clasificación de la Unión Internacional Contra el Cáncer (UICC) del año 2002, se establece una diferencia entre T1a (hallazgo de tumor incidental en $5 \%$ o menos del tejido resecado) y T1b (hallazgo de tumor incidental en más del $5 \%$ del tejido resecado). ${ }^{4}$ Esto se basa en el riesgo de progresión, que es sólo de $5 \%$ a los 5 años (aunque el 50\% progresa entre 10 y 13 años) para T1a; en cambio, la mayoría de los casos de T1b progresan a 5 años. ${ }^{15}$ Esto permite clasificar, por su agresividad potencial, como tumor "insignificante" al primero y tumor "clínicamente significativo" al segundo. ${ }^{16}$

Algunos autores informan una incidencia real muy baja de tumores transicionales puros $(0.6 \%)$ y se consideran que no es de utilidad realizar toma de biopsias en esta área, por el contrario, Ploussard reporta $19 \%$ de tumores de la zona de transición exclusivamente. ${ }^{17}$

Aunque los urólogos reconocen que el Cáncer Prostático identificado durante la revisión de biopsias por Resección Transuretral de Próstata es una presentación poco común en la época actual, la literatura no ha examinado a fondo la razón de este hallazgo; esta técnica quirúrgica no es considerada una herramienta para él diagnóstico de adenocarcinoma de próstata. Su importancia clínica radica en ser conveniente para los pacientes, hay que explicar durante el consentimiento informado del riesgo que existe de identificar carcinoma en el estudio histopatológico.

\section{Referencias.}

1. Secretaría de Salud. Programa de Acción; Cáncer de Próstata, México, D.F. Primera Edición, 2001; 9-20.

2. Hermanek P. TNM atlas. llustratec guide of the TNM/pTNM classification of malignant tumours. Springer-Velarg. Berlin. Edición 1997 (4ª):263326.

3. Alluano C. Incidental prostatic cancer: T1a-T1b. Our experience after observation/radical surgery and literature review; ActasUrol Esp. 2006;30(8):749-753. 
4. Berner A. Follow-up of localized prostate cáncer, with emphasis on previos undiagnosed incidental cancer. BJU International 1999;83-47.

5. Cheng L, Bergstralh EJ, Scherer BG. Predictors of cancer progression in T1a prostate adenocarcinoma. Cancer 1999; 85(6):1300-1304.

6. Lowsley O. "The development of the human prostate gland with reference to the development of other structures at the neck of the urinary bladder". Am J Anat. 1912; 13:299-349.

7. Lujan Galan M.Prevalence and clinical meaning of focal and incidental prostate cancers. ActasUrol Esp. 2007;31(8):819-824 .

8. Lee LS, Thiruneelakandasivam S.Are Transrectal Prostate Biopsies Routinely Indicated in $\mathrm{Pa}$ tients with Incidentally Diagnosed Prostate Cancer following Transurethral Resection of the Prostate for Benign Disease? Urol Int 2013;91:397-403.

9. Jophy V, Priya MK, Nisarg M.Incidentally detected adenocarcinoma prostate in transurethral resection of prostate specimens:a Hospital Based Study from India. Asian Pc J Cancer Prev. 2016;17(4):2255-2258.

10. Kien T Mai.Incidental Prostatic Adenocarcinomas and putative premalignant lesions in TURP specimens collected before and after the introduction of prostrate specific antigen screening. ArchPatholLab Med.2000;124:1454 -1456.
11. Dellavedova T, Ponzano R. Cáncer de Próstata como hallazgo en resección transuretral. Arch Esp Urol 2010;63(10): 855-861.

12. Margerber MJ, Andersen JT et al. Prostate volumen and serum prostate-specific antigen as predictors of acute urinary retention. Combined experience from three multinational placebo-controlled trials. Eur Urol. 2000;38: 563-568.

13. Puppo P. Repeted negative prostate biopsies with pesistently elevated or rising PSA: a modern urologic dilema. Eur Urol, 2007;52:639-641.

14. Steuber T, Karakiewcz PI. Transition zone cancers undermine the predictive accuracy of Partin table stage predictions. J Urol, 2005;173:737741.

15. Heidendreich A, Aus G. Guidelines on Prostate Cancer, p.33. Eur A Urol. Guidelines, 2009 edition.

16. Epstein JI. Can insignificant prostate cancer be predicted preoperatively in men with stage $\mathrm{T} 1$ disease? Semin Urol Onco. 1996;14(3):165-173.

17. Ploussard G, Dubosq F. Extensive biopsies and transuretral prostate resection in men with previous negative biopsies and high or increasing prostate specific antigen. $\mathrm{J}$ Urol, 2009;182(4):1342-1349. 